\title{
Sistema de medios de enseñanza tecnológicos de neumática y automatización para ingenieros
}

\begin{abstract}
RESUMEN
El vertiginoso avance de la ciencia y tecnología han facilitado la automatización de procesos productivos, motivando a que el sector industrial demande de profesionales técnicos con sólidos conocimientos en esta área. Este trabajo presenta un sistema de medios tecnológicos para la enseñanza y desarrollo de habilidades en el área de neumática y automatización. El sistema de medios consiste en tres fases categorizadas en temas, cada una de las cuales utiliza software de diseño y simulación, equipos didácticos y soporte en entornos virtuales de aprendizaje, que guian al estudiante desde los conocimientos básicos del principio de la neumática hasta el desarrollo de procesos automatizados. Este sistema de medios de enseñanza tecnológica se fundamenta en la acción, la experiencia, el grupo de trabajo y las aplicaciones prácticas, es decir aprender haciendo. Como resultado, se ha logrado mejorar el interés y motivación de los estudiantes hacia la neumática, la automatización y la formación de habilidades que le facilitan su inserción en el mercado laboral en esta importante área del sector industrial.
\end{abstract}

Palabras clave: Medios de enseñanza tecnológicos, neumática, automatización.

TECHNOLOGY TEACHING MEDIA SYSTEM OF PNEUMATIC AND AUTOMATION FOR ENGINEERS

\section{ABSTRACT}

The rapid advancement of science and technology has facilitated the automation of production processes, encouraging the industry to demand professionals with solid technical knowledge in this area. This paper presents a system of technological means for teaching and skills development in the area of pneumatic and automation. The media system consists of three phases categorized into themes, each of which uses design and simulation software, training and support teams in virtual learning environments, which guide the student from basic knowledge of the principle of pneumatics to the development of automated processes.

This system of technological teaching aids based on the action, experience, work groups and practical applications which means learning by doing. As a result, it has improved the interest and motivation of students to the pneumatics and the formation of skills that will facilitate their integration into the labor market in this important area of industry.

Keywords: Media education, pneumatics, automation.

\section{INTRODUCCIÓN}

Según el Instituto de Ingenieros Industriales, en inglés Institute of Industrial Engineers (IIE), la Ingeniería Industrial es la rama de las ingenierías encargada del análisis, interpretación, comprensión, diseño, programación y control de sistemas productivos y logísticos con miras a gestionar, implementar y establecer estrategias de optimización con el objetivo de lograr el máximo rendimiento de los procesos de creación de bienes y/o la prestación de servicios. Bajo este marco la Neumática y Automatización Industrial son herramientas interdisciplinarias de conocimientos que forman parte de su currículo y perfil profesional, cuyo propósito es la integración de técnicas y tecnologías de control con miras a una producción y/o gestión competente, segura y calificada.

Estudios realizados por Ocampo (2008), revelan el hecho de que un número considerable de estudiantes confrontan dificultades para la comprensión, asimilación, interpretación y aplicación a situaciones concretas los conocimientos relativos a diferentes tópicos de la Neumática.

La Neumática constituye una herramienta muy importante dentro del control automático en la industria. La automatización industrial por medio de componentes neumáticos, es una de las soluciones más sencillas, rentables y con mayor futuro de aplicación en procesos de producción y su finalidad es aumentar la competitividad, por lo que requiere de nuevas tecnologías.

La Neumática, tiene aplicación en sectores industriales como: alimentación, ensamblaje y manipulación, sistemas robotizados o industrias de procesos continuos, que son automatizadas, en gran parte neumáticamente, debido a las ventajas que esta presenta: elasticidad, velocidad de los actuadores, es una técnica limpia, simplifica enormemente la mecánica. Quizás la principal desventaja es que se requiere de una inversión añadida por la automatización y el mantenimiento constante del estado del aire.

1 Magíster en Gestión de la Producción, Doctor en Ingeniería Industrial, Docente Investigador Titular de la Facultad de Ingeniería de la Universidad Nacional de Chimborazo, Riobamba, Ecuador.

E-mail: pvillacres@unach.edu.ec

2 Magister en Diseño, Gestión y Evaluación de Proyectos Sociales y Productivos, Doctor en Ingeniería Industrial, Docente Investigador Titular de la Facultad de Ingeniería de la Universidad Nacional de Chimborazo, Riobamba, Ecuador E-mail: mcabrera@unach.edu.ec

3 Magister en Diseño, Gestión y Evaluación de Proyectos Sociales y Productivos, Doctor en Ingeniería Industrial, Docente Investigador Titular de la Facultad de Ingeniería de la Universidad Nacional de Chimborazo, Riobamba, Ecuador E-mail: hsalazar@unach.edu.ec 
Entre los conocimientos que los estudiantes de Ingeniería y especialmente de Industrial, comúnmente presentan mayores dificultades, se encuentran los concernientes a los fundamentos físicos (Mecánica de Fluidos), Elementos Neumáticos de Trabajo y Mando, Desarrollo de Circuitos de aplicación, Simulación de funcionamiento, Automatización Electro neumática. (Pieri y Diamantini, 2014).

Esta situación puede atribuirse a distintas causas, vinculadas tanto a la enseñanza como al aprendizaje, entre las cuales cabe señalar que no existe formación en Neumática que preceda a la universidad, esto se corrobora mediante la revisión de los contenidos en el currículum de los estudiantes de nivel medio en colegios de ciencias, a excepción de aquellos denominados técnicos cuya formación no siempre resulta la más adecuada; además el no alcanzar el sólido dominio en los principios fundamentales de los procesos de momento, calor y masa, los cuales se constituyen en premisas para el aprendizaje del resto de contenidos de la Neumática.

Si un estudiante es provisto con medios para experimentar y aplicar la teoría al mundo real, no sólo mejora la comprensión de los conceptos teóricos, sino también le da confianza para enfrentar situaciones complejas. (Torres y Redondo, 2006). El currículo en las carreras de formación técnica es objeto de constante perfeccionamiento debido al incesante desarrollo tecnológico, lo cual supone una Neumática y Automatización aplicada al sector productivo de avanzada, y en este caso específico desde la ingeniería industrial. Ello puede constituirse en un factor adverso si prevalecen métodos tradicionales de enseñanza de la misma.

Una alternativa para romper con los esquemas conductistas, tradicionales de enseñanza de la Neumática puede ser el perfeccionamiento de los métodos, la utilización de sistemas de medios de enseñanza tecnológicos adecuados, para lograr que los alumnos se apropien de la esencia del conocimiento a fin de aplicarla de manera creadora en la construcción de nuevos saberes, formación de habilidades y en la solución de problemas propios de la carrera.

En el desarrollo de circuitos neumáticos aplicados, se evidencia otro problema que conlleva a que los estudiantes rechacen estos temas, se refiere al cálculo y diagramación de una gran cantidad de circuitos en forma manual, sin opción a probar su funcionamiento, fiabilidad, posibles fallos y sin el apoyo de medios, entornos, recursos informáticos y equipos, que posibiliten la experimentación y aplicación real de los mismos.
Hay que tener en cuenta, además, que generalmente se produce aprendizaje a partir de una combinación de múltiples factores como son: la motivación, la activación de los conocimientos previos, las actividades de aprendizaje, por la selección de los materiales o recursos de aprendizaje fundamentados en una adecuada estrategia docente, las habilidades, los procesos, las actitudes, el entorno de interacción, la orientación, la reflexión y la evaluación). (Dondi, Sangrà \& Guàrdia 2000). Las Investigaciones pedagógicas relacionadas con la aplicación de medios tecnológicos en la enseñanza de la neumática, se fundamentan en la introducción de tecnologías de la información y comunicación en entornos virtuales y presenciales, software utilitario, dispositivos de laboratorio que faciliten los procesos de aprendizaje, cálculo, diseño, control, simulación y fiabilidad.

El objetivo del presente trabajo ha sido la creación de actividades de aprendizaje apoyadas en software de simulación y diseño (Festo Fluid Sim), integradas con prácticas de laboratorio y entornos virtuales de aprendizaje, bajo la concepción del B-learning (Blended learning), pedagógicamente diseñados para que el alumno profundice el objeto de estudio a su propio ritmo de aprendizaje. Es importante mencionar que la integración de este sistema de medios tecnológicos para la enseñanza promueve la construcción de conocimientos basados en el contexto social en donde se desenvuelven los alumnos, genera motivación hacia el aprendizaje, promueve el desarrollo de habilidades y destrezas, (Ausubel, Novak \& Hanesian 2003), así como la identificación de componentes, la creación de circuitos, la resolución de problemas inherentes a la neumática y automatización, así como una permanente interacción grupal y docente, que facilita la profundización de temas y mejora del perfil de ese profesional técnico en esta área.

\section{MATERIALES Y MÉTODOS}

Se seleccionó estudiantes universitarios que cursaban Neumática y Automatización en la Carrera de Ingeniería Industrial de la Universidad Nacional de Chimborazo, en Ecuador, los cuales fueron divididos en dos grupos de 25, a uno se aplicó el Sistema de Medios de Enseñanza Tecnológicos durante seis meses. Con el otro grupo se trabajó con la metodología de aprendizaje tradicional, considerando los efectos de este trabajo como grupo de control.

\section{APLICACIÓN DE LA METODOLOGÍA}

Este sistema de medios de enseñanza tecnológicos, propone tres fases en su ejecución: Neumáti- 
ca, Electroneumática y Control, Entorno Virtual de Aprendizaje. Cada una de las fases, se desarrolló de manera presencial combinada con un Entorno Virtual de Aprendizaje (EVA) bajo un ambiente B-learning, es decir un compartido entre la educación presencial con soporte virtual (Dalsgaard \& Paulsen, 2009), entorno centrado en el estudiante que además de ofrecer escenarios interactivos, eficaces y fácilmente accesibles y distribuidos, sin las limitaciones espacio-temporales del aprendizaje presencial. En este sistema se aborda una introducción al tema a tratar, se identifican componentes y herramientas fundamentales que intervienen en el tema estudiado, así como diagramas y esquemas del problema propuesto, coherentemente estructurados de tal manera que el aprendizaje y desarrollo de habilidades se consiguen a través de actividades a nivel de tema, en las cuales el estudiante aprende el principio de funcionamiento de los elementos de un circuito neumático o electro neumático realizando simulaciones con software profesional, para posteriormente complementar la actividad académica con montajes experimentales prácticos en el laboratorio con equipos didácticos para el efecto. Finalmente se complementa la clase presencial con actividades inherentes a los temas tratados en un entorno virtual de aprendizaje.

La evaluación final del curso se hace a través de la asignación de un proyecto en el cual el alumno debe reproducir un proceso industrial asignado por el profesor, utilizando todos los sistemas de medios estudiados y publicarlo en el entorno virtual de aprendizaje.

Durante el desarrollo del curso se planifican giras de observación a empresas productivas que disponen de procesos en donde se involucran sistemas neumáticos y de control que forman parte de la maquinaria y equipos analizados, así como a organizaciones que dan servicio y mantenimiento en esta área.

\section{Fase Neumática}

En la mencionada universidad, se utilizó el programa profesional Festo Fluid Sim, para desarrollar procesos de simulación y diseño de circuitos, (neumáticos, eléctricos), para la programación lógica, el software del programador lógico computarizado de Siemens Step 7, y Logo 230 RC. En esta primera fase se estudiaron los temas concernientes a fundamentos físicos de la Neumática, preparación y distribución del aire comprimido, cálculos básicos de compresores. Luego se les enseñó algunos elementos neumáticos de trabajo y mando, como cilin- dros, válvulas distribuidoras $3 / 2$ y $5 / 2$ vías, válvulas antirretorno, selectoras y de simultaneidad, válvula reguladora de presión, unidades de mantenimiento, reguladores de caudal, simbología normalizada, tipos de diagramas y esquemas. Las tareas asignadas a cada grupo de estudiantes se desarrollaron con técnicas de aprendizaje colaborativo.

La Figura 1 muestra un ejemplo de una tarea propuesta en esta primera fase. Básicamente una vez que el estudiante conoce el funcionamiento del software de simulación, y los elementos neumáticos de mando que intervienen en el circuito propuesto, él o el grupo de estudiantes son motivados a pensar cómo lograr que estos elementos neumáticos sean empleados para configurar el proceso de control asignado. Esta tarea consistió en que los estudiantes configuren la secuencia de dos cilindros de doble efecto (A y B). La Figura 1a y Figura 1b muestran el esquema realizado por el grupo de estudiantes utilizando el software de simulación de Festo Fluid Sim, en donde se aprecia una primera aproximación digital a lo que sería el funcionamiento del circuito con cada componente previo su montaje en el laboratorio. El profesor durante todo el proceso se convierte en un guía de aprendizajes, se determina la importancia de paradas de emergencia (válvula de $3 / 2$ vías, elemento Z2) para detener el proceso ante cualquier eventualidad, y los arranques propios del sistema. Todos los grupos realizan simulaciones distintas, sobre la marcha se incluyen válvulas reguladoras de caudal (elementos AO2, $\mathrm{AO} 3, \mathrm{BO} 2, \mathrm{BO} 3$ ), para controlar la velocidad de los actuadores e interiorizar su efecto en el circuito y demás elementos que permiten el ciclo automático de la tarea desarrollada.

Realizada la simulación y evaluado el funcionamiento correcto del circuito por los estudiantes con la guía del profesor se procedió a realizar el montaje experimental. Se observó cómo, una vez completado el mismo, los estudiantes exploran elementos neumáticos, su funcionamiento, lo que conlleva a que el proceso sea lo más real e industrial posible. La compañía FESTO, posee un KIT de dispositivos, software para el diseño de esquema de los circuitos, tablas, diapositivas que facilitaron los montajes y experiencias prácticas. Finalizada la experimentación práctica se discutió y comparó todos los montajes, de modo que con el debate se establecen los resultados de aprendizaje.

Terminadas las tareas intraula los estudiantes, complementaron el tema tratado desarrollando las actividades planteadas en el EVA (Entorno Virtual de Aprendizaje), cuyo detalle se abordará en ésa fase de investigación. 
a

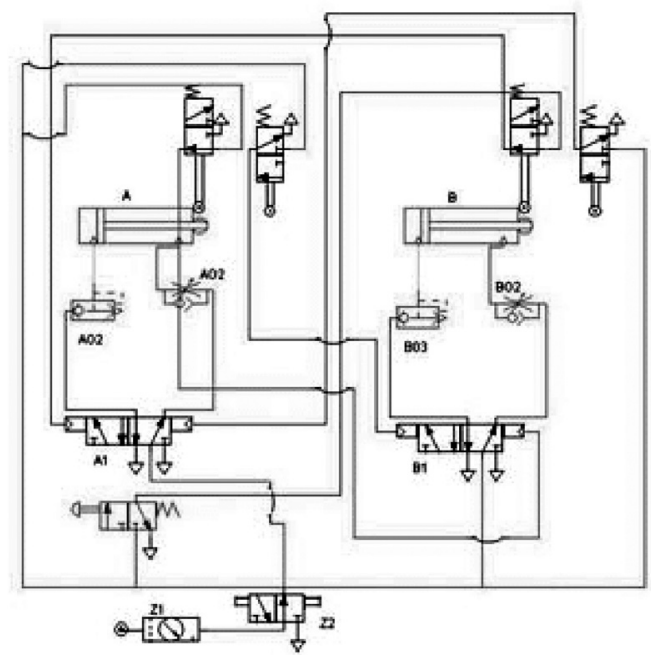

b

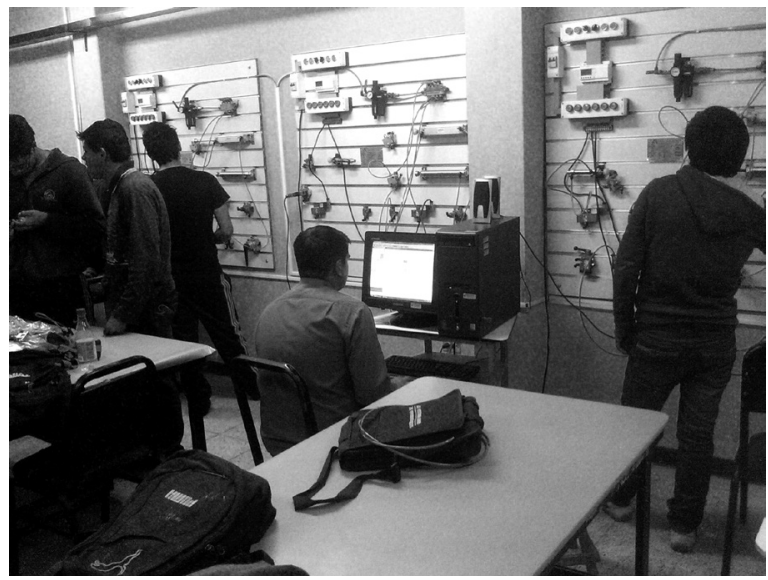

Fuente: Elaboración propia.

Figura 1. Diseño, simulación y montaje en laboratorio de secuencia de dos cilindros por estudiantes.

\section{Fase de Electroneumática y Control}

La Neumática pura, significa trabajar con cantidad de conexiones para lograr un proceso, lo cual a nivel industrial es una desventaja. Los avances de la ciencia han permitido la creación de nuevos elementos que pueden ser controlados por la Electrónica, y las empresas se han adaptado a los cambios en esta área.

En la Electroneumática los actuadores siguen siendo neumáticos, los mismos que en la Neumática básica, pero las válvulas de gobierno mandadas neumáticamente son sustituidas por electroválvulas activadas con electroimanes en lugar de pilotadas con aire comprimido. Las electroválvulas son convertidores electroneumáticos que transforman una señal eléctrica en una actuación neumática. Por otra parte los sensores, fines de carrera y captadores de información son dispositivos eléctricos que se usaron para la regulación y la automatización. Las ventajas de la Electroneumática sobre la Neumática pura se concretaron en la capacidad que tienen la Electricidad y la Electrónica de emitir, combinar, transportar y secuenciar señales, que las hacen extraordinariamente idóneas para cumplir tales fines.

En esta fase fue necesario que los estudiantes tengan como conocimientos previos los fundamentos de la Electricidad y Electrónica aplicada, el funcionamiento de relés, temporizadores, sensores y la lógica de conexión cableada. Dado que su dominio permitió introducir las técnicas de automatización por programadores lógicos controlables PLC, sobre los mismos esquemas de circuitos electro neumáticos previamente construidos por símbolos eléctricos y determinar su utilidad e importancia en máquinas y equipos dentro de los procesos.

La Figura 2 muestra una práctica realizada por el grupo de estudiantes utilizando elementos de accionamiento neumáticos y control eléctrico del montaje. En esta tarea los educandos van desarrollando aplicaciones industriales. En toda máquina o proceso automatizado se deben tener en cuenta variables como: alarmas, paradas de emergencia, varias opciones de mando (accionamiento manual, automático, periódicos, etc.), la Electrónica permite realizar todos estos controles de forma más eficiente. Los estudiantes realizaron los mismos montajes hechos en tareas anteriores con elementos solamente neumáticos y al comparar descubren la importancia y beneficio de la Electroneumática. En la Figura 2a, se muestra el control de un cilindro de doble efecto con sensores inductivos, en donde se aprecia el uso de relés auxiliares, contactos, pulsadores y elementos de señalización. En este tema los alumnos aprendieron a realizar diagramas de control de maquinaria, con lógica de relés. La simulación y posterior experiencia práctica hizo que los estudiantes tengan confianza en sus propias habilidades adquiridas y que estén satisfechos con los resultados de sus experiencias de aprendizaje. Una vez que los alumnos interiorizan y dominan estos conocimientos y habilidades, es mucho más sencillo integrar a los mismos circuitos su control por Controlador Lógico Programable (Programmable Logic Controller) (PLC), a través de diagramas por 
lógica de contactos ladder o KOP (Figura $2 b$ ), por bloques de funciones (FUB), Texto estructurado $u$ Organigrama secuencial de funciones (SFC). Son los estudiantes los que construyen un montaje con los dispositivos existentes en el laboratorio, este montaje consta de elementos neumáticos y eléctricos que son controlados por un dispositivo lógico programable (PLC) trabajando con distintas señales de entradas (analógicas o digitales) y salidas por relé, transistorizadas o por optoacoplador. Aplicando la metodología secuencial para la solución, de los problemas propuestos que empiezan con el diagrama espacio-fase donde deciden si utilizaran un control a lazo abierto o cerrado, luego con el mé- todo Grafcet definieron el control de etapas y transiciones. Para luego diseñar un programa por lógica ladder (KOP) o por bloques de funciones (FUP). Este programa lo simularon en el computador y en la etapa final del proyecto calibrarán y ajustarán los componentes en dependencia del tipo de circuito desarrollado.

La evaluación de esta fase tuvo un carácter cualitativo y cuantitativo con énfasis en los procesos y esta se realiza por medio del planteamiento de un proyecto neumático en donde se apliquen los conocimientos adquiridos para resolver un problema industrial dentro del contexto social en el que se desenvuelven los estudiantes. a

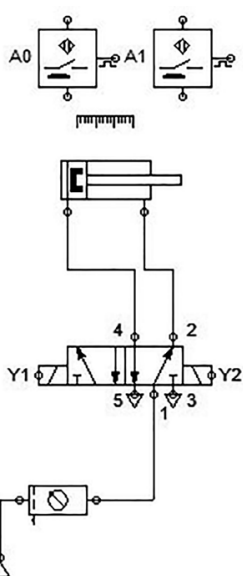

b

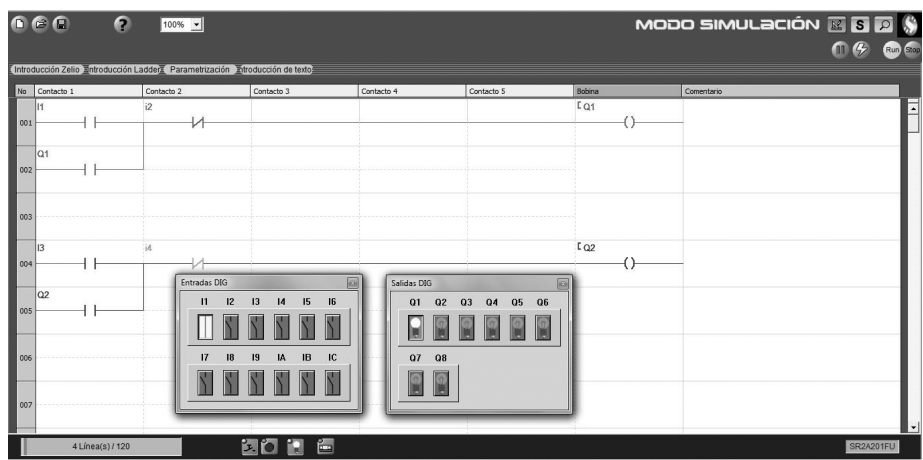

Fuente: Elaboración propia.

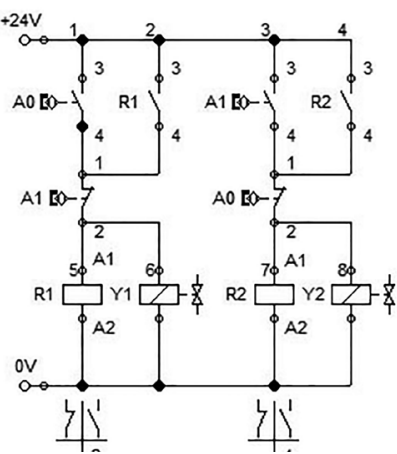

Figura. 2 Diseño, simulación y montaje electro neumático.

\section{Fase entorno virtual de aprendizaje}

Esta fase consiste en integrar las aulas virtuales en la educación presencial creando un entorno virtual de aprendizaje bajo el enfoque del B-learning es decir un compartido entre la educación presencial con soporte virtual, esto es el aprendizaje, facilitado a través de la combinación eficiente de diferentes métodos de impartición, modelos de enseñanza y estilos de aprendizaje, y basado en una comunicación transparente de todas las áreas implicadas en el curso. El EVA (Figura 3) se creó sobre la plataforma Moodle, y comprendió un conjunto de recursos y actividades que involucraron el uso de tecnologías de la información y comunicación para complementar y profundizar el proceso de enseñanza aprendizaje de la neumática. Su éxito radicó en elaborar el EVA con criterio Pedagógico, estructurado en concordancia con los temas tratados presencialmente, generando complementos competentes a los temas tratados sin duplicar contenidos y con un peso académico del 40 al $50 \%$ en su evaluación como parte del curso.

El aula virtual se dividió por bloques en la plataforma Moodle, estructurándose con los siguientes bloques:

Bloque cero, en el cual se incluyó la sección de información con la Guía para iniciar el curso de Neumática, una corta presentación y una rúbrica de evaluación. La sección de comunicación que arrojó una cartelera en línea en donde se publicaron las novedades del evento. La sección de interacción, con cafetería virtual, un espacio de socialización informal entre el maestro y los alumnos que tomen el curso de neumática y un taller para orientar a los alumnos en las actividades programadas y brindar el apoyo técnico, tecnológico e informático.

El Bloque académico con una sección de exposiciones en donde se mostró los recursos y contenidos programáticos usando libros digitales, slideshares, 
videos, documentos en Word, PDF, entre otros. La sección de actividades que involucró la sección de rebote con consultas, chats, videoconferencias o glosario de términos técnicos, construcción por medio de wikis, foros y talleres y comprobación en donde el profesor verificó si se consolidó el conocimiento a través de trabajos individuales o grupales cuestionarios, pruebas, proyecto final. Bloque de cierre en el cual retroalimentamos lo estudiado y se establece un acuerdo para la evaluación y coevaluación del curso con fines de promoción y mejoramiento del EVA.

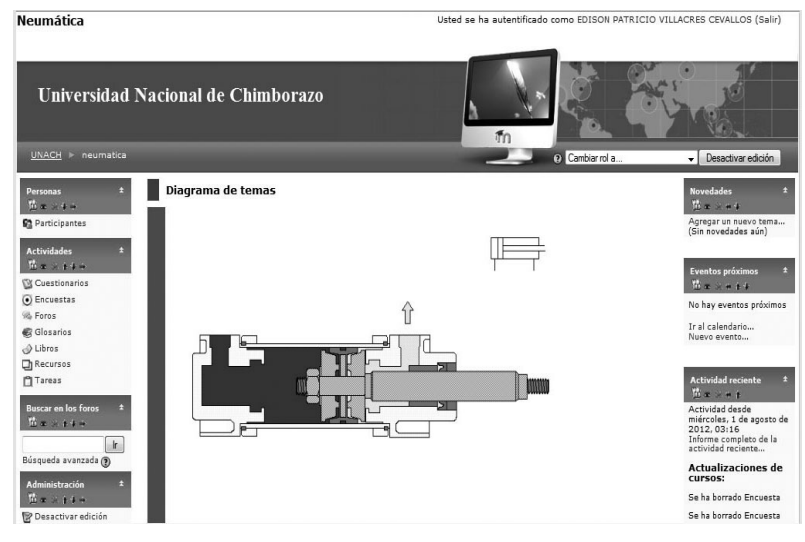

Fuente: Elaboración propia.

Figura 3. Entorno virtual de aprendizaje para la enseñanza de la neumática y automatización.

\section{Actividades Complementarias}

De acuerdo a Allen \& Seaman (2010) es necesario conceptualizar y visualizar el conocimiento teórico adquirido en la práctica para construir el nuevo conocimiento, por esta razón al final del curso, se programó una gira de observación a la empresa TUBASEC, dedicada a lo producción de techos en fibrocemento, en lana de vidrio y polipropileno, con la finalidad de que los estudiantes establezcan analogías, identifiquen elementos, procesos, interioricen y refuercen los conocimientos adquiridos así como su pertinencia en ambientes reales.

\section{ANÁLISIS ESTADÍSTICO}

El análisis estadístico fue realizado aplicando estadística descriptiva e inferencial se usó el módulo de encuesta predefinida de la plataforma Moodle, incluyendo COLLES (Constructivist On-Line Learning Environment Survey) y ATTLS (Attitudes to Thinking and Learning Survey), útiles en la evaluación y estimulación del aprendizaje. El programa estadístico Informático SPSS $₫$ Statistics 23.0, la prueba de bondad de ajuste Kolmogorov-Smirnov, permitió determinar la normalidad de los datos. Con la prueba t para muestras independientes, se verificó la varianza de rendimiento de los grupos investigados con un nivel de significancia $\alpha=0.05$

\subsection{Resultados}

La prueba Z Kolmogorov-Smirnov, para la muestra investigada como procedimiento de bondad de ajuste, permitió medir el grado de concordancia existente entre la distribución del conjunto de datos y la distribución teórica específica, considerando los 50 estudiantes incluido el grupo de control, el grado de significancia asintótica bilateral dio como resultado 0.149 , valor mayor al alfa de 0.05 , por tanto la distribución fue normal.

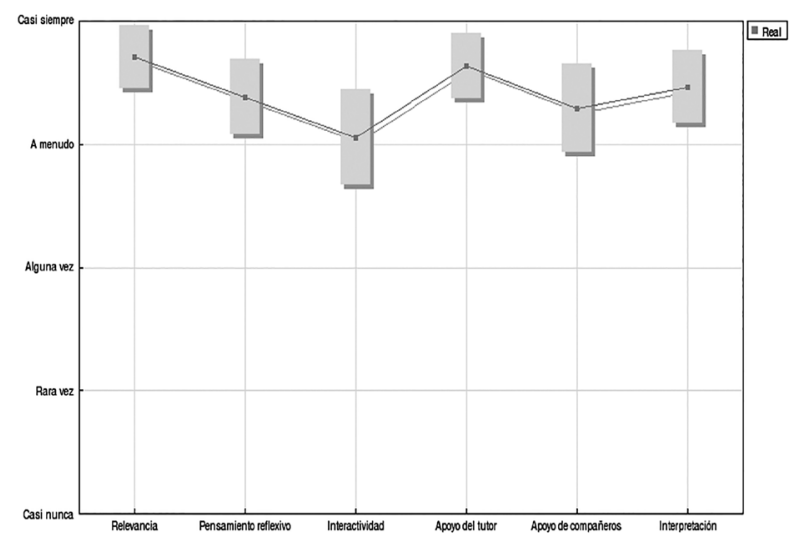

Fuente: Elaboración propia.

Figura 4. Prueba de colles. Relevancia en aprendizaje de neumática y automatización.

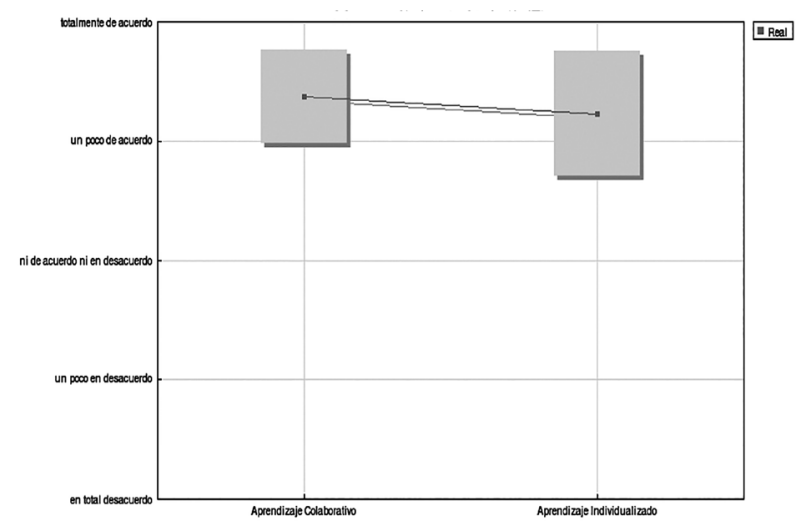

Fuente: Elaboración propia.

Figura 5. Prueba ATTLS. Actitud hacia el Aprendizaje de Neumática y Automatización. 
Tabla 1. Prueba T para Muestras Independientes considerando la variable Rendimiento Académico.

\begin{tabular}{|c|c|c|c|c|c|}
\hline \multicolumn{6}{|c|}{ Estadisticos de grupo } \\
\hline & Grupos & $\mathrm{N}$ & Media & $\begin{array}{c}\text { Desviación } \\
\text { tip. }\end{array}$ & $\begin{array}{l}\text { Error tip. de la } \\
\text { media }\end{array}$ \\
\hline Rendimiento & Grupo Control & 25 & 5,64 & .810 & .162 \\
\hline & $\begin{array}{l}\text { Grupo Sistema de } \\
\text { Medios }\end{array}$ & 25 & 7,84 &, 850 & 170 \\
\hline
\end{tabular}

\begin{tabular}{|c|c|c|c|c|c|c|c|c|c|c|}
\hline \multicolumn{11}{|c|}{ Prueba de muestras independientes } \\
\hline & & \multicolumn{2}{|c|}{$\begin{array}{l}\text { Prueba de Levene para la } \\
\text { igualdad de varianzas }\end{array}$} & \multicolumn{7}{|c|}{ Prueba $T$ para la igualdad de medias } \\
\hline & & \multirow[b]{2}{*}{$\mathrm{F}$} & \multirow[b]{2}{*}{ Sig. } & \multirow[b]{2}{*}{$t$} & \multirow[b]{2}{*}{ gl } & \multirow[b]{2}{*}{ Sig. (bilateral) } & \multirow{2}{*}{$\begin{array}{l}\text { Diferencia de } \\
\text { medias }\end{array}$} & \multirow{2}{*}{$\begin{array}{l}\text { Error tip. de la } \\
\text { diferencia }\end{array}$} & \multicolumn{2}{|c|}{$\begin{array}{c}95 \% \text { Intervalo de confianza para } \\
\text { la diferencia }\end{array}$} \\
\hline & & & & & & & & & Inferior & Superior \\
\hline \multirow[t]{2}{*}{ Rendimiento } & $\begin{array}{l}\text { Se han asumido } \\
\text { varianzas iguales }\end{array}$ & \multirow[t]{2}{*}{.332} & \multirow[t]{2}{*}{.567} & $-9,364$ & 48 & .000 & $-2,200$ & .235 & $-2,672$ & $-1,728$ \\
\hline & $\begin{array}{l}\text { No se han asumido } \\
\text { varianzas iguales }\end{array}$ & & & $-9,364$ & 47,888 & .000 & $-2,200$ & .235 & $-2,672$ & $-1,728$ \\
\hline
\end{tabular}

Resumen del procesamiento de los casos

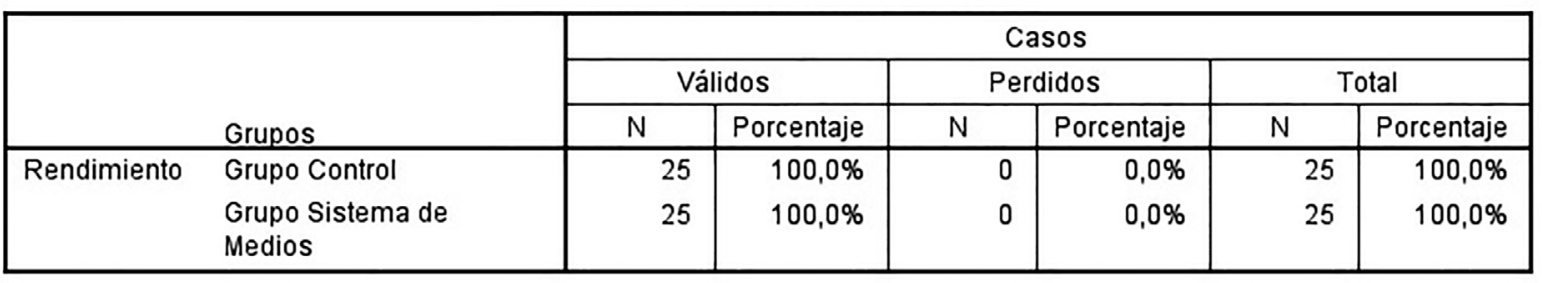

Fuente: Elaboración propia. Resultados obtenidos con SPSS 23.0.

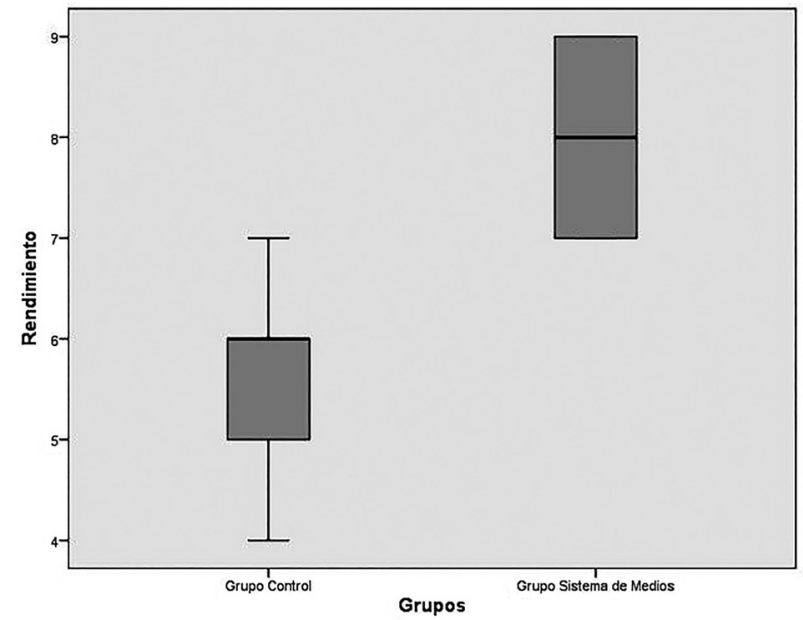

Fuente: Elaboración propia.

Figura 6. Diagrama de caja. Rendimiento por Grupos investigados.

\section{DISCUSIÓN}

En el análisis de impacto de la investigación, el sistema de medios identifica la variable dependiente; el aprendizaje, mismo que fue validado por las pruebas de COLLES y ATTSL, la hipótesis de in- vestigación permitió comprobar si la aplicación de este sistema de medios en efecto mejoraba el rendimiento de los estudiantes de Ingeniería Industrial en Neumática y Automatización a través de una prueba $\mathrm{T}$, con un nivel de significancia .

Los resultados iniciales son sumamente alentadores, pues los alumnos aprendieron a trabajar en grupo en un esquema colaborativo, de forma autónoma, desarrollaron competencias en manejo de tecnologías de la información y comunicación, diseño y simulación de circuitos complejos, detección de fallas, mantenimiento y reparación, todo esto con una visión global de aplicabilidad a automatización de procesos productivos en la empresa.

La introducción de los sistemas de medios de enseñanza tecnológicos en las aulas está poniendo en evidencia una nueva definición de roles, especialmente, para los alumnos y docentes. Los primeros, gracias a estos, están adquiriendo mayor autonomía y responsabilidad en el proceso de aprendizaje, lo que obliga al docente a salirse de su rol clásico como única fuente de conocimiento. Su uso ha generado transversalidad, nuevos ambientes de Aprendizaje y necesidad de formación continua.

Al aplicar el módulo de encuesta de COLLES (Constructivist On-Line Learning Environment Sur- 
vey) apreciamos en el polígono de frecuencias resultante (Figura 4), como el Grupo de estudiantes que trabajó con el sistema de medios tecnológicos para la enseñanza de la Neumática y Control, a menudo y Casi siempre generó procesos constructivistas con relevancia, pensamiento reflexivo, interactividad, así como apoyo del tutor y compañeros, favoreciendo la interpretación de tareas y actividades propuestas en este ambiente de aprendizaje en línea.

La prueba ATTLS (Attitudes to Thinking and Learning Survey), de la Figura 5, demuestra una clara tendencia a estar totalmente de acuerdo en que el sistema de medios tecnológicos genera actitudes hacia el pensamiento y aprendizaje Colaborativo e Individualizado.

El programa estadístico SPSS Statistics 23.0, con la prueba t para muestras independientes permitió comprobar la hipótesis de esta investigación, mostrando en estadísticas de grupo (Tabla 1), una media en el rendimiento académico validado sobre 10 puntos; de 5.64 para el grupo de control respecto al 7.84 del grupo, que trabajó con esta metodología propuesta, evidenciando un incremento significativo en la media del rendimiento académico estudiantil.

La prueba de Levene para la igualdad de varianzas, arroja un nivel de significancia de 0,567, mismo que es mayor al impuesto en la investigación , ello conlleva entonces que, en la Prueba T para la Igualdad de Medias el Sig o nivel de significancia bilateral sea de $\mathbf{0 . 0 0 0}$, mismo que al ser menor que $\mathbf{0 , 0 5}$, demuestra que la varianza de la variable grupo de control es diferente del grupo al cual se aplicó la metodología del Sistema de Medios Tecnológicos para la Enseñanza de la Neumática y Automatización. Comprobándose de esta forma que si existe mejora significativa en el rendimiento académico estudiantil al aplicar la propuesta motivo de esta investigación.

El diagrama de caja de la Figura 6, suministra información sobre la distribución de la variable rendimiento, respecto de los grupos investigados, en donde claramente se aprecia que no existen valores atípicos bajos ni altos, una mediana de 6 y 8 respectivamente. Al aplicar el Rango Intercuartílico (RIC), RIC = percentil 75 - percentil 25, se obtienen los siguientes resultados:

RIC para el grupo de control es de $6-5=1$, RIC para el grupo sistema de medios es de $9-7=2$, comprobándose un incremento significativo en el rendimiento académico.

\section{CONCLUSIONES}

- El sistema de medios tecnológicos mejoró la enseñanza y el rendimiento académico de los estudiantes de Neumática y Automatización en Ingeniería Industrial de la Universidad Nacional de Chimborazo, incrementándose desde $5.64 / 10$ hasta una media de $7.84 / 10$, resultando un aporte innovador en el lugar donde se realizó la investigación, sin desmerecer que esta propuesta puede ser utilizada no solamente en Ingeniería Industrial, sino en aquellas carreras cuyo perfil profesional involucre la enseñanza de Neumática y Automatización.

- El proceso docente con tareas y actividades prácticas proporciona la oportunidad a estudiantes y profesores para construir conocimientos efectivos. Permite a su vez desarrollar habilidades en el manejo de software profesional y educativo de simulación, manejo de instrumentación, elementos neumáticos y programación de procesos, en fin, el estudiante logra una formación completa del área de estudio.

- La integración de sistema de medios de enseñanza tecnológicos, permite que los alumnos analicen y entiendan el principio de funcionamiento de todos los componentes que intervienen en la neumática y automatización, motivo de estudio a través del uso de laboratorios para el montaje de circuitos previamente simulados, la aplicación de tecnologías de la información y comunicación soportadas en ambientes virtuales de aprendizaje para complementar la actividad presencial y desarrollar las habilidades necesarias en el profesional de la educación técnica.

- Tomar en cuenta la estructura académica de un ambiente virtual de aprendizaje con una metodología pedagógica en el tratamiento del proceso educativo. que faciliten la construcción del conocimiento.

- Se debe considerar que la actividad virtual que se realice debe tener una validación académica, esta debe ser un complemento competente, con criterios de evaluación claros e importantes para las actividades presenciales y virtuales.

- Utilizar los recursos Web, Redes sociales, sistemas LMS, Metaverso, para crear el ambiente virtual de aprendizaje creativo, que apoyados de estrategias pedagógicas, organizan los aprendizajes en base a interacciones e interactividad entre los miembros del entorno. 
- Las giras de observación facilitan la vinculación de la teoría con la práctica en el sector industrial y de servicios.

\section{REFERENCIAS BIBLIOGRÁFICAS}

[1] Ausubel, D., Novak, J. \& Hanesian (2003), Psicología Evolutiva: Un punto de vista Cognoscitivo. 15a Reimpresión, México: Editorial Trillas.

[2] Allen, I. E., \& Seaman, J. (2010). Learning on demand: Online education in the United States, 2009. Sloan Consortium. PO Box 1238, Newburyport, MA 01950.

[3] Dondi, C., Sangrà, A., \& Guàrdia, L. (2000). Proyecto BENVIC, una metodología y criterios de calidad para evaluar entornos y plataformas virtuales de aprendizaje. Catalunya, España.

[4] Dalsgaard, C., \& Paulsen, M. F. (2009). Transparency in cooperative online education. The
International Review of Research in Open and Distributed Learning, 10(3). Recuperado de http://dx.doi.org/10.19173/irrodl.v10i3.671

[5] Ocampo, J. (2008). Implementando Estrategias de Aprendizaje Combinado a la Enseñanza de Cursos de Ingeniería en UNITEC. Documento procedente del VI International Latin American and Caribbean Conference for Engineering and Technology, Tegucigalpa, Honduras.

[6] Pieri, M., \& Diamantini, D. (2014). An e-learning web 2.0 experience. Procedia-Social and Behavioral Sciences, 116(1), 1217-1221. Recuperado de http://dx.doi.org/10.1016/j.sbspro.2014.01.371

[7] Torres, J. y Redondo, J. (2006). Reparación y Automatización de una Máquina Universal de Ensayos. Procedente del VI congreso nacional de ingeniería mecánica, Universidad de Los Andes, Mérida, Venezuela. 
